\title{
The Effect of Physical Aging on the Time-Decay Behavior of Second Harmonic Generation Activity in a Poled Polymer Film
}

\author{
Sang Cheol Lee, * Akira Kidoguchi, Toshiyuki Watanabe, Hironobu Yamamoto, \\ Takeshi Hosomi, and Seizo MiYata \\ Chemical and Biological Science and Technology, \\ Tokyo University of Agriculture and Technology, \\ Nakamachi, Koganei-shi 184, Japan \\ (Received October 19, 1990)
}

\begin{abstract}
The efficient second harmonic generation has been observed in poled polymer systems. But, the nonlinear optical activity of these systems was relaxed with time. In order to enhance the stability of second harmonic activity over time, poling and subsequent physical aging process was applied to a doped polymer system. The aging process changes the relaxation behavior and improves the temporal stability of the second harmonic activity.
\end{abstract}

KEY WORDS Second Harmonic Generation / Physical Aging / Poling /

Relaxation /

Many organic materials for nonlinear optical applications have been investigated because of their flexibility of artificial molecular design and large optical nonlinearities as compared with those of inorganic crystals. ${ }^{1,2}$

Recently, it has been reported that efficient second harmonic generation (SHG) can be observed in poled polymer systems such as molecularly doped or covalently functionalized glassy polymer. ${ }^{3,4}$ In these systems, a structural non-centrosymmetry, which is a prerequisite for accessing the SHG effect, is offered by the alignment of nonlinear optical chromophores in polymer matrix. The polar chromophores are oriented by appling electric field above glass transition temperature, from the original random orientation to the direction of electric field. However, these systems show the decay of SHG activity with the lapse of time, even below glass transition temperature. This undesirable phenomenon is caused by the disorientation of ordered chromophores in poly- mer matrix.

In this study, poly(4-vinyl pyridine-costyrene) was doped with $N, N^{\prime}$-bis(4-nitrophenyl)-methanediamine (NMDA) of which the calculated molecular hyperpolarizability $\beta$ is $12.2 \times 10^{-30}$ e.s.u. The doped film was poled above glass transition temperature $\left(T_{\mathrm{g}}\right)$ and subsequently aged just below $T_{\mathrm{g}}$. And the effect of physical aging on the relaxation of SHG activity over time was investigated with varying the aging time.

\section{EXPERIMENTAL}

Poly(4-vinyl pyridine-co-styrene) (10 wt $\%$ styrene) was purchased from Aldrich Co. and purified by dissolving in methanol followed by filtration and precipitation into water. The precipitated copolymer was dried in vacuum oven at $100^{\circ} \mathrm{C}$ for 3 days. Molecular weight measurement was carried out in a GPC chromatograph (Waters) with DMF as eluent,

* Present address: Department of Polymer Science and Engineering, Kum-Oh Institute of Technology, Kumi, Kyung Buk 730-070, Korea. 
relative to polystyrene standard $\left(M_{n}=4.2 \times\right.$ $10^{5}, M_{w}=7.8 \times 10^{5}$ and polydispersity $=1.9$ ).

NMDA was synthesized by the reaction of $p$-nitroaniline with formaldehyde in methanol at room temperature. The chemical structure was verified by ${ }^{1} \mathrm{H}$ NMR spectroscopy as follows:<smiles>O=[N+]([O-])c1ccc(NCCNc2ccc([N+](=O)[O-])cc2)cc1</smiles>

NMDA shows 80 times stronger SHG than urea in powder test, even though the crystal structure has not determined yet.

The polymer films containing nonlinear chromophore NMDA were prepared by spin-coating on preheated $\left(80^{\circ} \mathrm{C}\right)$ ITO glass substrate from DMF solutions, and dried in vacuum oven at $100^{\circ} \mathrm{C}$ for at least $30 \mathrm{~h}$. The film thicknesses were measured by a surface profiler (DEKTAK-11-AV type). Film thicknesses were $7-9 \mu \mathrm{m}$ and thickness variation of a film was $0.2 \mu \mathrm{m}$.

$T_{\mathrm{g}}$ 's of the copolymer and the mixtures with NMDA were measured by Rigaku thermal analyzer (CN 8085EI). The $T_{\mathrm{g}}$ almost linearly decreases with increasing the NMDA content as shown in Figure 1. The NMDA plays a role of a low-molecular-weight diluent and consequently enhances the mobility of the system.

The film was first corona-poled by applying

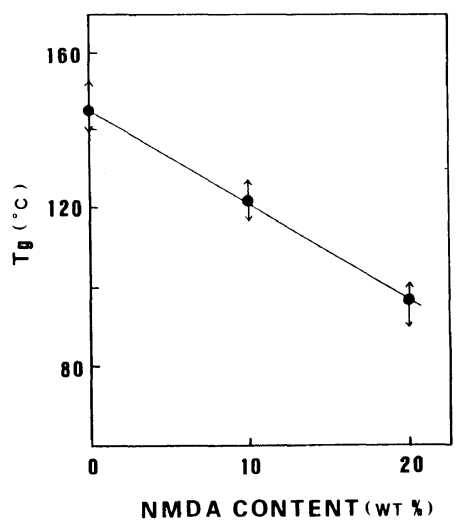

Figure 1. Glass transition temperature as a function of NMDA content. electric field at about $5^{\circ} \mathrm{C}$ above $T_{g}$ for $30 \mathrm{~min}$, and then cooled down to room temperature in the presence of electric field. In the subsequent aging process, the poled film was kept at about $5^{\circ} \mathrm{C}$ below for a finite time. During the poling and aging process, silicon oil was coated on the film surface to avoid the film breakdown, and $4 \mathrm{kV}$ electric field was applied between ITO anode and pin-shaped cathode which was spaced about $1 \mathrm{~cm}$ from the film surface.

The SHG measurements were performed at room temperature with lapse of time, by illuminating a Nd-YAG laser with a $1064 \mathrm{~nm}$ wavelength.

\section{RESULTS AND DISCUSSION}

Assuming that a poled polymer film belongs to poing group $\infty \mathrm{mm}$, the second harmonic polarization $P$ induced by electric field $E$ is given by ${ }^{5}$

$$
\begin{aligned}
& P_{\mathrm{x}}^{2 \omega}=2 d_{15} E_{\mathrm{x}} E_{\mathrm{z}} \\
& P_{\mathrm{y}}^{2 \omega}=2 d_{15} E_{\mathrm{y}} E_{\mathrm{z}} \\
& P_{\mathrm{z}}^{2 \omega}=d_{31} E_{\mathrm{x}}^{2}+d_{31} E_{\mathrm{y}}^{2}+d_{33} E_{\mathrm{z}}^{2}
\end{aligned}
$$

where $d_{i j}$ are the second harmonic coefficients. Since Kleinman symmetry ${ }^{6}$ gives $d_{15}=d_{31}$, two nonzero coefficients $\left(d_{31}\right.$ and $\left.d_{33}\right)$ remain, which are responsible for the generation of second harmonic wave.

The second harmonic coefficients were determined by Maker fringe analysis. ${ }^{7}$ In this method, the second harmonic intensity for uniaxial materials is given by

$$
\begin{aligned}
P_{2 \omega}= & \left(512 \pi^{3} / A\right) t_{\omega}^{4} T_{2 \omega} d^{2} P(\theta)^{2} P_{\omega}^{2} \\
& \times\left[1 /\left(n_{\omega}^{2}-n_{2 \omega}^{2}\right)^{2}\right] \sin ^{2} \psi(\theta)
\end{aligned}
$$

where $P_{\omega}$ and $P_{2 \omega}$ are the incident fundamental and the transmitted second harmonic powers at an angle $\theta$ from the film normal, $A$ the area of the beam, $t_{\omega}$ and $T_{2 \omega}$ transmission factors, $d$ the nonlinear coefficient, $P(\theta)$ the projection factor, $n_{\omega}$ and $n_{2 \omega}$ the refractive indices at the fundamental and second harmonic frequencies, and $\psi(\theta)$ the angular dependence of second 
harmonic power. The second harmonic coefficient $d_{33}$ was calculated from the angular dependence of $p$-polarized transmitted second harmonic intensity against $p$-polarized incident wave, assuming that $d_{31}=d_{33} / 3 .^{8}$ The value was compared to $d_{11}$ of quartz. Typical Maker fringe of a sample is shown in Figure 2.

The refractive indices (Table I) of a film were obtained using prism coupling technique, ${ }^{9}$ with changing the wavelength of laser. Argon and Helium-Neon lasers were used. The coupling of a laser beam by a prism into a planar wave guide is governed by the angle of incidence of the light onto the prism base. We could observe strong coupling of light into the prism at three

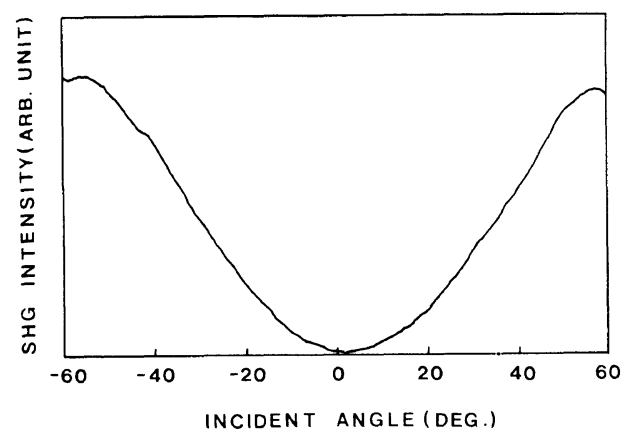

Figure 2. Second harmonic intensity for a typical film containing $20 \mathrm{wt} \%$ NMDA as a function of incident angle of the $1064 \mathrm{~nm}$ beam. angles. The refractive indices of the fundamental and the second harmonic wave were calculated by a nonlinear regression of Sellmeir equation, which is given by

$$
n^{2}=A+\frac{B}{1-C / \lambda^{2}}-D \lambda^{2}
$$

The calculated refractive index data were $n_{2 \omega}=1.633$ and $n_{\omega}=1.601$.

Figure 3 shows the $d_{33}$ decay at room temperature as a function of time after poling and aging. The second harmonic coefficient for poled and aged films (B and $\mathrm{C}$ in Figure 3) decays more slowly and the residual second harmonic activity is stronger than that for a simply poled film (A in Figure 3). For chromophore-doped polymer films, it is considered that the higher the degree of orientation

Table I. Values of refractive indices as a function of wavelength of a film containing $20 \mathrm{wt} \% \mathrm{NMDA}$

\begin{tabular}{cc}
\hline$\lambda / \mathrm{nm}$ & $n$ \\
\hline 476.5 & 1.662 \\
488.0 & 1.653 \\
496.5 & 1.649 \\
514.5 & 1.639 \\
632.8 & 1.624 \\
\hline
\end{tabular}

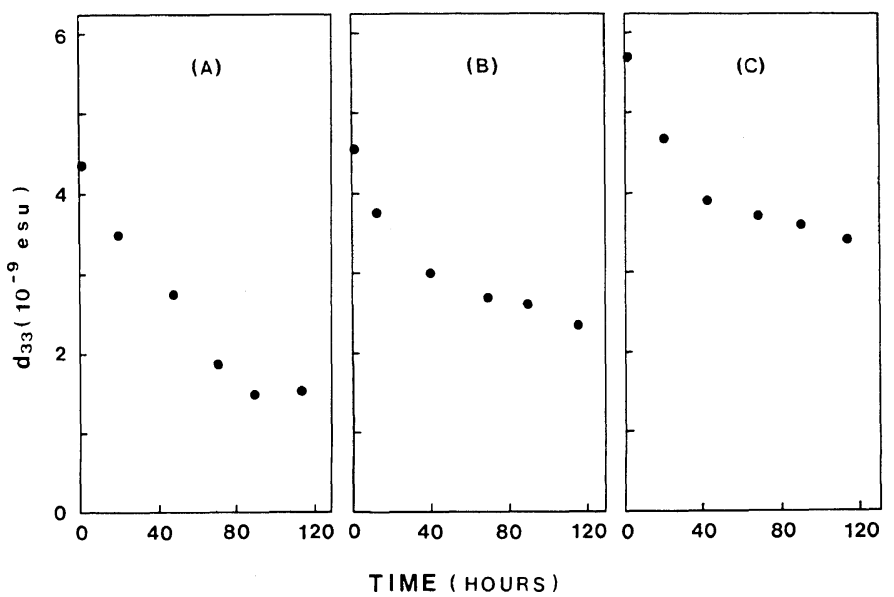

Figure 3. Time decay of the second harmonic coefficient for films containing $20 \mathrm{wt} \%$ NMDA: (A) $0 \mathrm{~h}$; (B) $2 \mathrm{~h}$; (C) $5 \mathrm{~h}$ aged samples. 
of chromophores, the faster the decay rate. However, the decay behavior of the film aged for $5 \mathrm{~h}$ is similar to that of the film aged for $2 \mathrm{~h}$, even though the absolute $d$ value of the former is higher than that of the latter. This means that the decay rate decreases with increasing the aging time. Two important origins, which influence the realignment of nonlinear chromophores to the random orientation, can be considered: one is the secondary relaxation of a glassy polymer, and the other is the inherent thermal mobility of the low-molecular-weight chromophore. The free volume of the system is an important factor to affect above two origins, because molecular motion in the bulk state can not take place without the presence of void volume. Usually, the free volume below $T_{\mathrm{g}}$ is larger than that in the equilibrium state, because a non-equilibrium state is frozen in during cooling of a polymer from above to below $T_{\mathrm{g}}$. The physical aging changes the distribution, the size, and the content of free volume in a non-equilibrium state. Therefore, the mobility of chromophore is constrained and the temporal stability of second harmonic efficiency is improved.

\section{REFERENCES}

1. D. J. Williams, Ed., "Nonlinear Optical Properties of Organic and Polymeric Materials," The American Chemical Society, Washington, D.C., 1983.

2. D. S. Chemla and J. Zyss, Ed., "Nonlinear Optical Properties of Organic Molecules and Crystals," Academic, New York, N.Y., 1987.

3. K. D. Singer, J. E. Sohn, and S. J. Lalama, Appl. Phys. Lett., 49, 248 (1986).

4. C. Ye, T. J. Marks, J. Yang, and G. K. Wong, Macromolecules, 20, 2322 (1987).

5. J. F. Nye, "Physical Properties of Crystals," Clarenden, Oxford, 1967.

6. D. A. Kleinman, Phys. Rev., 126, 1977 (1962).

7. J. Jerphagnon and S. K. Kurtz, J. Appl. Phys., 41, 1667 (1970).

8. C. Ye, N. Minami, T. J. Marks, J. Yang, and G. K. Wong, Macromolecules, 21, 2899 (1988).

9. R. Ulrich and R. Torge, Appl. Opt., 12, 2901 (1973). 